\title{
Femoral Hernia: A Review of the Clinical Anatomy and Surgical Treatment
}

\author{
Makio Mike", Nobuyasu Kano \\ Department of Surgery, Kameda Medical Center, Kamogawa, Japan \\ Email: \#mmike@ace.ocn.ne.jp
}

Received September 2, 2013; revised October 1, 2013; accepted October 9, 2013

Copyright (C 2013 Makio Mike, Nobuyasu Kano. This is an open access article distributed under the Creative Commons Attribution License, which permits unrestricted use, distribution, and reproduction in any medium, provided the original work is properly cited.

\begin{abstract}
Purpose: Femoral hernia is a kind of ventral hernia that surgeons commonly encounter, second in frequency only to inguinal hernia. Femoral hernias often require emergency surgery because of incarceration or strangulation of the intestine. In addition, intestinal resection may need to be considered based on intestinal viability. Definitive preoperative diagnosis and strategic planning for surgery are thus important. The surgeon should consider the operation in the context of the clinical anatomy of the abdominal cavity. Therefore the essence of the clinical anatomy and treatment of femoral hernia is described. Methods: The medical records of 38 patients who underwent femoral hernia repair between March 2006 and November 2011 were retrospectively analyzed. Results: Femoral hernioplasty was performed with original mesh repair or Ruggi's repair plus iliopubic tract repair (or Bassini's repair). The mean patient age was 76.7 years, and a female predominance was apparent. Twenty-four patients underwent emergency surgery with a diagnosis of incarcerated femoral hernia. Nine patients showed intestinal strangulation and underwent resection of the small intestine. Four patients developed complications. One patient died due to aspiration pneumonia. No recurrences were encountered after 6 months to 6 years of follow-up. Conclusion: Femoral hernia is an important surgical condition with high rates of incarceration/strangulation and intestinal resection. Correct preoperative diagnosis of femoral hernia and a strict operative strategy are important. The original mesh repair is effective and easy to perform.
\end{abstract}

Keywords: Femoral Hernia; Clinical Anatomy; Mesh Repair; Ruggi’s Repair

\section{Introduction}

Femoral hernia is a ventral hernia commonly encountered by surgeons in clinical practice, although less common than inguinal hernia. Femoral hernia often needs an emergency operation because of incarceration or strangulation [1]. In addition, intestinal resection may need to be considered after taking into account the viability of the intestine. A definitive preoperative diagnosis and strategic plan for surgery are thus important. The choice of operation should be considered based on the clinical anatomy of the abdominal cavity. Since an understanding of the anatomy of this region is comparable to an understanding of the basic factors behind the clinical anatomy of the entire abdominal cavity, the essence of the clinical anatomy and treatment of femoral hernia are described.

\footnotetext{
*Conflicts of interest: The authors declare that they have no potential conflicts of interest, including specific financial interests, relationships or affiliations relevant to the subject matter or materials discussed in the manuscript.

"Corresponding author.
}

\section{Anatomy of the Inguinal and Femoral Region}

The basic format of the peritoneal configuration of the body walls in fetal life is shown in Figure 1. Basic interpretations of the fascial composition of the body circumference have been provided by Tobin et al. [2] and Satoh [3]. According to these interpretations, the structure of the body below the diaphragm can be simplified as a straight intestine within a cylindrical body. The basis of body composition can then be divided into the composition of the peritoneal cavity (composition in the cylinder) and the composition of the body wall (composition of the cylinder wall). In the former, the cranial abdomen includes the dorsal and ventral mesentery, while the caudal abdomen includes only the dorsal mesentery involving the intestine. The latter has a ringed composition, with the body walls symmetrical in relation to the central position of the muscle layer (Figure 1). The trunk has typically been regarded as an onion-like, multi-layered structure [4]. Corresponding to the subcutaneous superfi- 


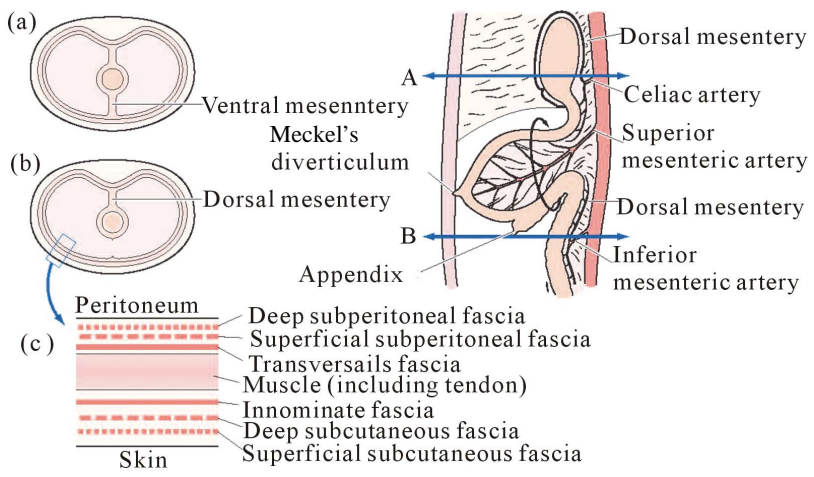

Figure 1. Basic structures of the abdominal wall and embryonic peritoneal cavity. The basis of body composition can then be divided into the composition of the peritoneal cavity and the composition of the body wall. The body walls are symmetrical in relation to the central position of the muscle layer.

cial fascia (Camper's fascia) and subcutaneous deep fascia (Scarpa's fascia), deep subperitoneal fascia and superficial subperitoneal fascia exist circumferentially around the abdominal wall (Figure 1).

Corresponding to the innominate fascia as an outer aponeurosis (investing fascia) along with the external oblique aponeurosis, the transversalis fascia (investing fascia) extends circumferentially around the abdominal wall as an inner aponeurosis with the transversus abdominis muscle. The transversalis fascia can thus be recognized as the endoabdominal fascia. The fascial structures present in the body wall are embryologically represented by the term superficial or deep, defined in relation to the skin surface. The terminology used throughout the body for anatomical terms should be used given the continuity of the abdominal cavity. It is also important to understand the differences between the concepts of fascia and muscle aponeurosis.

The orifice of the femoral hernia, called the femoral ring, is bordered laterally by the femoral vein, anteriorly by the iliopubic tract, and medially by Cooper's ligament [5] (Figure 2). The iliopubic tract represents a thickening of the transversalis fascia, and the transversalis fascia should extend to the femoral canal and further to Cooper's ligament according to the concepts proposed by Tobin et al. [2] and Satoh [3]. The transversalis fascia is originally considered fragile, but this aponeurosis is thought to become toughened by intertwining fibers from the transversus abdominis aponeurosis and the internal oblique aponeurosis. In the posterior wall of the inguinal canal, three layers can reportedly be separated: the internal oblique aponeurosis; transversus abdominis aponeurosis; and transversalis fascia [6].

\section{Materials and Surgical Method}

The majority of cases of femoral hernias involve elderly women, but here, the more complex procedure for men is described.

The start of surgery is the incision along the inguinal ligament. The superficial and deep fasciae of the subcutaneous tissue are incised to reach the innominate fascia. By peeling back the innominate fascia toward the oval fossa, the underlying viscera are exposed, covering the superior and deep subperitoneal fasciae, transversalis fascia and peritoneum (the term sac represents all fasciae for convenience) (Figure 3). Here, the inguinal canal is exposed with an incision into the external oblique fascia, and the spermatic cord is secured and taped in the region of the pubic tubercle. An incision is made from the internal inguinal ring to the pubic tubercle. The neck of the sac should be seen wrapped in the superficial and deep subperitoneal fasciae and the peritoneum (Figure 4). Here, the sac is dissected from the iliopubic tract and Cooper's ligament. Lateral dissection is not performed at this point. The transversalis fascia is incised between the iliopubic tract and Cooper's ligament using Kelly forceps, and the femoral ring takes on a triangular shape (Figure

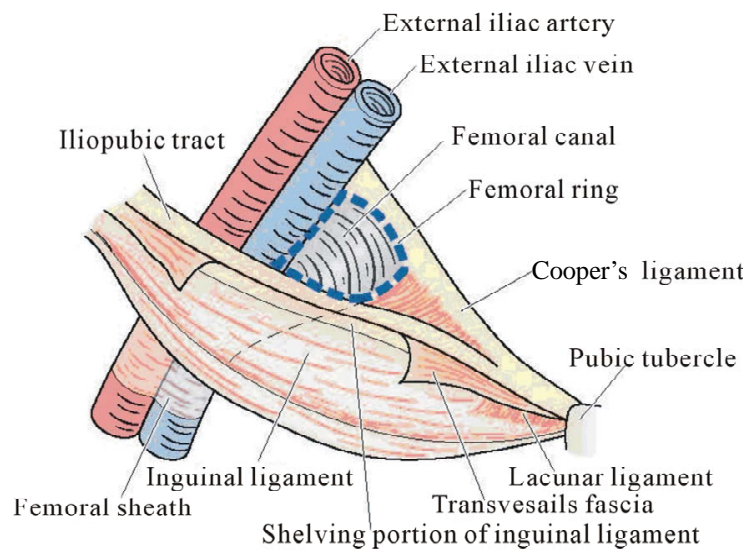

Figure 2. Anatomy around the femoral ring. The femoral ring is structured with the iliopubic tract, Cooper's ligament and the medial border of the external iliac vein.

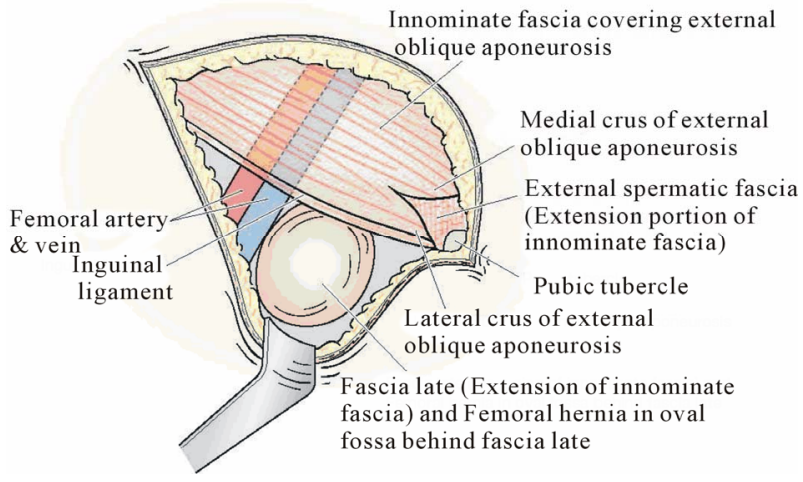

Figure 3. Innominate fascia and fascia lata. The innominate fascia is dissected caudally from the groin. A hernial sac can be confirmed covered with superficial and deep subperitoneal fascia and transverse fascia. 


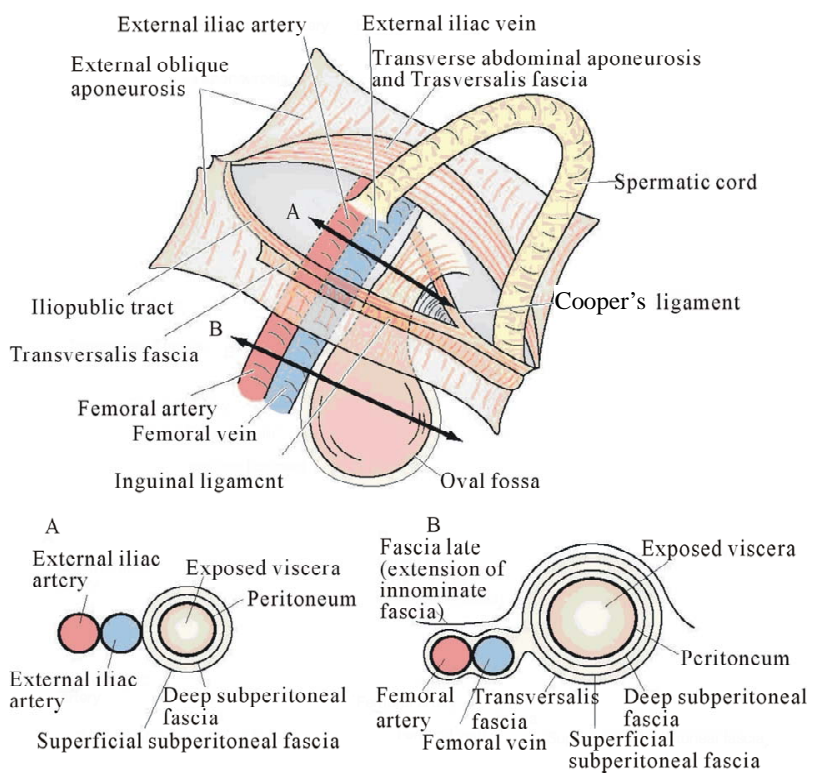

Figure 4. Cross-section of the cranial and caudal sides of the fascia. An incision is made into the posterior wall of the inguinal canal from the internal ring to the pubic tubercle. The neck should be seen as a sac wrapped in superficial and deep subperitoneal fascia. The relationship between the fascia is shown in the cranial (A) and caudal (B) portions of the inguinal ligament.

5(a), arrow). Opening the superficial subperitoneal fascia introduces a space between the superficial and deep subperitoneal fasciae on the medioventral side, and dissection of this space allows the neck of the sac to be taped (Figure 5). Fatty tissue is present in the space between the superficial and deep fasciae, and the space is relatively loose to dissect, allowing easy taping of the neck of the sac. In this taping, blunt-tipped forceps with a long, strong curvature are convenient to use. Holding a Nelaton's catheter or cotton tape through the neck of the sac, reduction of the sac to the abdominal side can be attempted. Alternatively, reduction of the sac to the abdominal side can be tried by compressing the sac at the oval fossa. If reduction of the sac to the abdominal side is impossible, incision of the deep subperitoneal fascia and peritoneum may be needed to open the sac at the superior portion of the iliopubic tract and confirm the contents. If peeling the sac at the oval fossa, five sheaths from the ventral side can also be dissected to reach the real sac (actual sac) as shown in Figure 4(b). A femoral hernia is not incarcerated or strangulated at the femoral ring, but is considered to be incarcerated and strangulated with stenosis of the sac itself. The sac thus needs to be opened and the constricted area eventually released.

In cases of suspected bowel necrosis due to strangulation of the intestine, the affected segment of the intestine is not resected immediately. Replacing the segment back into the peritoneal cavity for 10 min with support from

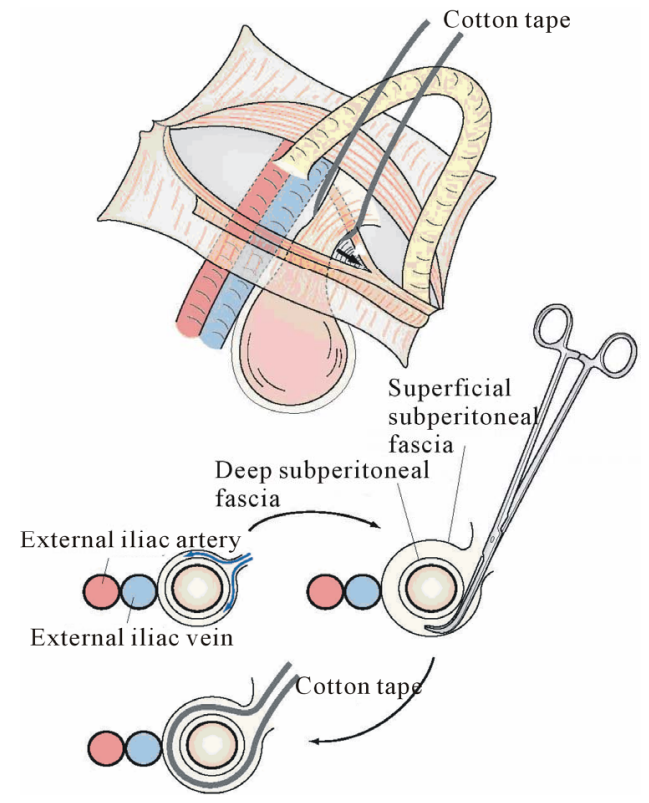

Figure 5. Dilatation of the femoral ring and method for taping the neck of the sac. The femoral ring is dilated with an incision in the transversalis fascia (A). The superior subperitoneal fascia is opened on the medioventral side, and dissection is performed between the superficial and deep subperitoneal fasciae from mediodorsal to laterodorsal. The neck of the sac can then be taped by performing the same procedure from ventral to lateral.

the seromuscular sutures of the intestine near good blood flow is preferable, and improvements can be obtained by paying attention to the color of the bowel.

The basic concept of surgical repair for femoral hernia is to cover the femoral ring and groin area with mesh [6]. Thus, a 7.6-cm $\times 15-\mathrm{cm}$ lightweight mesh (Prolene Soft $\AA$; ETHICON, Inc., Somerville, NJ, USA) is used. First the mesh is sutured to Cooper's ligament and folded to overlap the pubic tubercle by about $1.5 \mathrm{~cm}$ and to ensure sufficient coverage of the supravesical hernia [7] (Figure 6(a)). The return line of the mesh is then sutured to the iliopubic tract or to the shelving portion of the inguinal ligament (Figure 6(b)). After folding the mesh, the posterior wall of the inguinal canal is covered as in Lichtenstein's repair, providing posterior support (Figure 6(c)) $[8,9]$.

If the surgery was contaminated, Ruggi's repair $[10,11]$ and anterior iliopubic tract repair [12] are performed. In other words, 1-0 nylon sutures are added between Cooper's ligament and the iliopubic tract or the shelving portion of the inguinal ligament from the pubic tubercle to close the femoral ring (Figure 7(a)), and then anterior iliopubic tract repair is performed as posterior reinforcement of the inguinal canal (Figure 7(b)). However, since excessive femoral ring closure has been reported to lead to the obstruction of the external iliac vein, care is required. 


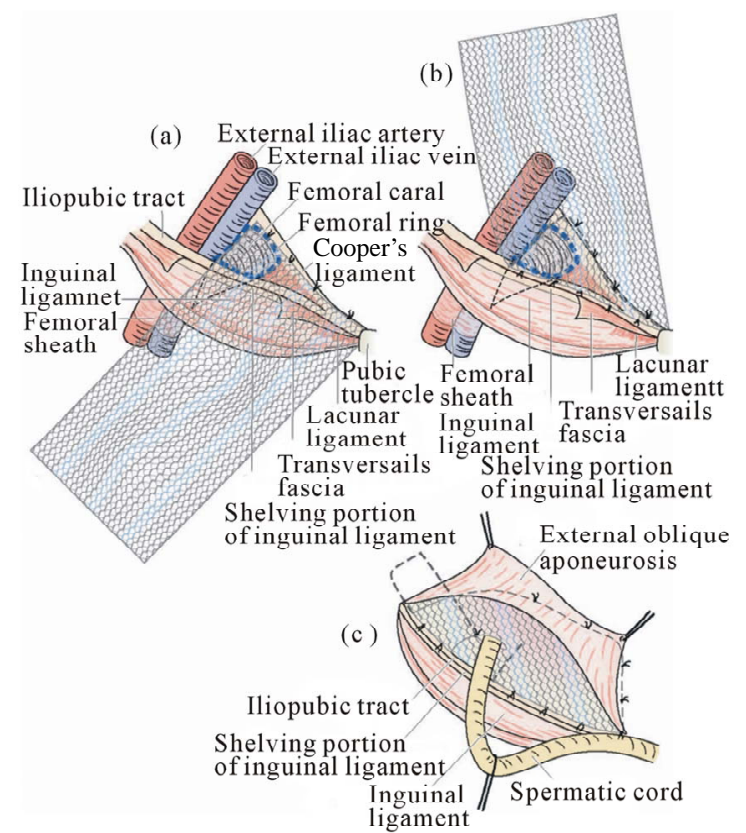

Figure 6. Femoral hernia repair in clean operation. (a) The narrow side of the mesh is sutured to Cooper's ligament; (b) The mesh is sutured to the iliopubic tract or shelving portion of the inguinal ligament; (c) The posterior wall of the inguinal canal is reinforced, as in Lichtenstein's repair.
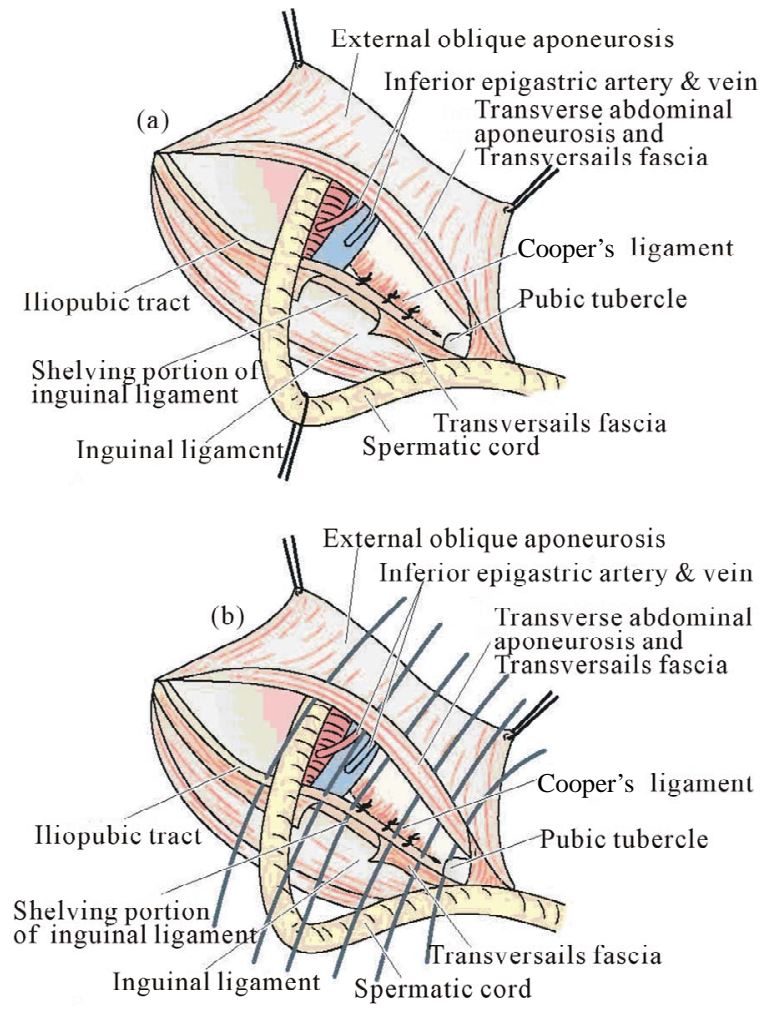

Figure 7. Femoral hernia repair in dirty operation. (a) Ruggi's repair: sutures between Cooper's ligament and the shelving portion of the inguinal ligament or the iliopubic tract from the inside; (b) After the above anterior iliopubic tract repair.

\section{Results}

Between March 2006 and November 2011, a total of 38 patients with femoral hernia underwent surgery using an inguinal approach in our institution. These patients included 29 women (76.3\%) and 9 men (23.7\%), with a mean age of 76.7 years (range, 53 - 99 years). Primary hernias accounted for $97.4 \%$ of cases (37 of 38), whereas only $2.6 \%$ of cases ( 1 of 38 ) was considered to represent recurrent femoral hernia after a femoral approach. Twenty-four patients (63.2\%) underwent emergency surgery with a diagnosis of incarceration or strangulation of femoral hernia. Nine patients showed strangulation and underwent resection of the small intestine. All resections were performed through the inguinal incision. First intraoperative inspection showed strangulated intestines with color change evident in the wall, changing to a viable coloration in 4 patients after about 10 - 15 min in the abdominal cavity. All patients underwent definitive surgery. Mesh repair was used in 30 patients (78.9\%), while 8 patients (21.1\%) were treated using Ruggi's repair with iliopubic tract repair or with Bassini’s repair.

Four patients developed complications (3 patients as emergencies). Two cases of complications were related to wound infection, and one involved peritonitis due to delayed perforation of the intestine that required re-operation with intestinal resection. Another had massive bloody ascitic fluid because of an impending rupture of an abdominal aortic aneurysm at the hernia operation and died due to aspiration pneumonia 1 month 5 days after operation. No recurrences were detected after 6 months to 6 years follow-up (average follow-up period, 9.7 months).

\section{Discussion}

Femoral hernia is thought to represent about 2\% - 4\% of inguino-femoral hernias [1,13-16], but it is often overlooked during inguinal hernia repair [15]. Many reports have described intraoperative identification of femoral hernia after preoperative misdiagnosis [17]. Accurate preoperative diagnosis of the hernia is important for achieving optimal surgical results.

The anatomy of femoral hernia is often stated incorrectly, and the terminology of the surrounding area for these hernias has not been strictly defined. An understanding restricted to the local anatomy of the inguinal and femoral regions is thus a limitation. Understanding the basic fascial composition of the body circumference as described by Tobin et al. [2] and Sato [3] clarifies the inguinal and femoral anatomy. As a matter of course, the pathologies involving this area also become more understandable.

For surgical procedures, hernia repair uses the principle of "broad support against pressure based on Pascal's 
law and minimal biological response”. We therefore used a lightweight mesh [18]. The mesh is required to cover the femoral ring, pubic tubercle, area of the supravesical hernia and the lateral triangle of the inguinal canal, which is an area that includes the internal inguinal ring and the tissues immediately lateral to it [19]. Our original procedure with mesh is simple and reliable for treating femoral hernia because of the certain closure of the femoral ring with mesh sheet and of the reinforcement of the inguinal floor, as in Lichtenstein's repair [8,9].

Furthermore, if surgery is contaminated, use of foreign material should be avoided. Many reports have recommended McVay's repair for such situations [20,21]. According to the intent of the original transition suture, the manipulation closes the angle between Cooper's ligament and the iliopubic tract and prevents protrusion through the femoral ring. However, stenotic complications can be caused by pressure to the femoral vein using this maneuver [22,23], so the procedure requires close attention. We adopted Ruggi's repair to close the femoral ring, which includes intermittent sutures between Cooper's ligament and the inguinal ligament [10,11], plus anterior iliopubic tract repair [12] as a non-mesh repair. Iliopubic tract repair is a reinforcement of the inguinal floor with sutures added between the transversus abdominal arch and the iliopubic tract. However, many elderly individuals already show a weakened aponeurosis fascia, including the transversus abdominis aponeurosis, so Ruggi's repair plus Bassini's repair [24] is recommended. Bassini's repair is the method of reinforcement of the inguinal floor with sutures between three layers (internal oblique muscle, transversus abdominal muscle and transverse fascia) and the inguinal ligament. In Ruggi's repair, temporary sutures should be used for the outermost suture ligation to ensure that the suture does not compress the femoral vein.

A unified strategy for the treatment of femoral hernia is needed and requires accurate preoperative diagnosis and surgical techniques based on clinical anatomy.

\section{Conclusion}

Femoral hernia is an important surgical pathology with high rates of incarceration/strangulation and intestinal resection. A correct preoperative diagnosis of femoral hernia and a strict operative strategy are important. The original mesh repair is effective and easy to perform.

\section{REFERENCES}

[1] U. Dahlstrand, S. Wollert, P. Nordin, G. Sandblom and U. Gunnarsson, "Emergency Femoral Hernia Repair. A Study Based on a National Register," Annals of Surgery, Vol. 249, No. 4, 2009, pp. 672-676.

http://dx.doi.org/10.1097/SLA.0b013e31819ed943
[2] C. E. Tobin, J. A. Benjamin and J. C. Wells, “Continuity of the Fascia Lining the Abdomen, Pelvis, and Spermatic Cord," Surgery, Gynecology \& Obstetrics, Vol. 83, No. 5, 1946, pp. 575-596.

[3] T. Sato, "Fundamental Plan of the Fascial Strata of the Body Wall,” Igakunoayumi, Vol. 114, No. 13, 1980, pp. C168-C175. (in Japanese)

[4] M. Mike and N. Kano, "Laparoscopic-Assisted Low Anterior Resection of the Rectum-A Review of the Fascial Composition in the Pelvic Space,” International Journal of Colorectal Disease, Vol. 26, No. 4, 2011, pp. 405-414. http://dx.doi.org/10.1007/s00384-010-1107-2

[5] T. H. Quinn, "Anatomy of the Groin: A View from the Anatomistm,” In: L. M. Nyhus and R. E. Condon, Eds., Hernia, 5th Edition, Lippincott Williams \& Wilkins, Philadelphia, 2002, pp. 55-70.

[6] R. Bendavid, “The Transversalis Fascia: New Observations,” In: R. Bendavid, J. Abrahamson, M. E. Arregui, J. B. Flament and E. H. Phillips, Eds., Abdominal Wall Hernia, Springer, New York, 2000, pp. 97-100.

[7] M. S. Kavic, “Chronic Pelvic Pain in Women,” In: R. Bendavid, J. Abrahamson, M. Arregui, J. B. Flament and E. H. Phillips, Eds., Abdominal Wall Hernias, SpringerVerlag, New York, 2001, pp. 632-638. http://dx.doi.org/10.1007/978-1-4419-8574-3_94

[8] I. L. Lichtenstein, A. G. Shulman, P. K. Amid and M. M. Montllor, "The Tension-Free Hernioplasty," The American Journal of Surgery, Vol. 157, No. 2, 1989, pp. 188193. http://dx.doi.org/10.1016/0002-9610(89)90526-6

[9] P. K. Amid, A. G. Shulman and I. L. Lichtenstein, "Critical Scrutiny of the Open "Tension-Free" Hernioplasty," The American Journal of Surgery, Vol. 165, No. 3, 1993, pp. 369-371. http://dx.doi.org/10.1016/S0002-9610(05)80847-5

[10] A. V. Moschcowitz, "Femoral Hernia: A New Operation for Radical Cure,” New York Medical Journal, Vol. 7, No. 10, 1907, p. 396.

[11] G. Ruggi, "Metado Operativo Meovo per la Cure Radicale Dell’Ernia Crurale,” Bull Sci Med Bologna, Vol. 7, No. 3, 1892, pp. 223-229.

[12] R. E. Condon, “Anterior Iliopubic Tract Repair,” In: L. M. Nyhus and R. E. Condon, Eds., Hernia, 3rd Edition, Lippincott, Philadelphia, 1978, pp. 195-211.

[13] M. Bay-Nielsen, H. Kehlet, L. Strand, J. Malmstrøm, F. H. Andersen, P. Wara, P. Juul and T. Callesen, "Quality Assessment of 26,304 Herniorrhaphies in Denmark: A Prospective Nationwide Study," Lancet, Vol. 358, No. 9288, 2001, pp. 1124-1128. http://dx.doi.org/10.1016/S0140-6736(01)06251-1

[14] G. Sandblom, S. Haapaniemi and E. Nilsson, "Femoral Hernias: A Register Analysis of 588 Repairs," Hernia, Vol. 3, No. 3, 1999, pp. 131-134. http://dx.doi.org/10.1007/BF01195312

[15] F. Glassow, "Femoral Hernia: Review of 2105 Repairs in a 17 Year Period,” The American Journal of Surgery, Vol. 150, No. 3, 1985, pp. 353-356. http://dx.doi.org/10.1016/0002-9610(85)90077-7

[16] T. Mikkelsen, M. Bay-Nielsen and H. Kehlet, "Risk of 
Femoral Hernia after Inguinal Herniorrhaphy,” British Journal of Surgery, Vol. 89, No. 4, 2002, pp. 486-488. http://dx.doi.org/10.1046/j.0007-1323.2002.02058.x

[17] M. Mike, N. Kano and K. Koh, "Femoral Hernia: Its Clinical Anatomy and Surgical Treatment," Rinshogeka, Vol. 63, No. 13, 2008, pp. 1763-1769. (in Japanese)

[18] Y. Watanabe, M. Mike and N. Kano, "Inguinal Hernia Repair in Consideration of Mesh Material,” Geka, Vol. 69, No. 11, 2007, pp. 1341-1344. (in Japanese)

[19] A. I. Gilbert, M. F. Graham and W. J. Voight, "The Lateral Triangle of the Groin,” Hernia, Vol. 4, No. 4, 2000, pp. 234-237. http://dx.doi.org/10.1007/BF01201073

[20] M. Mike and N. Kano, "Inguinofemoral Hernia Repair in the Original Papers-McVay Operation,” Shujyutu, Vol.
61, No. 13, 2007, pp. 1939-1943. (in Japanese)

[21] C. B. McVay, "Groin Hernioplasty: Cooper's Ligament Repair,” In: L. M. Nyhus and R. E. Condon, Eds., Hernia, 2nd Edition, JB Lippincott, Philadelphia, 1978, pp. 179193.

[22] E. Y. Normington, D. P. Franklin and S. I. Brotman, "Constriction of the Femoral Vein after McVay Inguinal Hernia Repair,” Surgery, Vol. 111, No. 3, 1992, pp. 343347.

[23] H. M. Nissen, "Constriction of the Femoral Vein Following Inguinal Hernia Repair,” Acta Chirurgica Scandinavica, Vol. 141, No. 4, 1975, pp. 279-281.

[24] E. Bassini, "New Operative Method for the Cure of Inguinal Hernia,” Ciné-Med Inc., Woodbury, 2008. 